\title{
Identify Unsuitable Patients with Left Main Coronary Artery Disease in Intermediate SYNTAX Scores Treated by Percutaneous Coronary Intervention
}

\author{
Chunxiao Zhang MD, ${ }^{1}$ Yaguang Zheng $\mathrm{PhD},{ }^{2}$ Xinbin Liu MD,${ }^{3}$ Yutong Cheng MD, ${ }^{4}$ Yang Liu MD, ${ }^{5}$ \\ Yan Yao MD, ${ }^{4}$ Xinguo Wang MD,${ }^{4}$ Jianping Xu MD ${ }^{6}$ \\ ${ }^{1}$ Department of Cardiovascular Surgery, Beijing Anzhen Hospital, Capital Medical University, Beijing, China; Department \\ of Cardiovascular Surgery, Peking University International Hospital; ${ }^{2}$ Connell School of Nursing, Boston College, Boston, \\ MA, USA; ${ }^{3}$ Department of Cardiovascular Surgery, Aerospace Center Hospital, Beijing, China; ${ }^{4}$ Department of Cardiology, \\ Beijing Anzhen Hospital, Capital Medical University, Beijing, China; ${ }^{5}$ Department of Cardiology, No. 305 Hospital of People's \\ Liberation Army (PLA), Beijing, China; ${ }^{6}$ Department of Cardiovascular Surgery, Fuwai Hospital, Chinese Academy of Medical \\ Sciences, National Center for Cardiovascular Diseases; Department of Cardiovascular Surgery, Peking University International \\ Hospital, Beijing, China
}

\section{ABSTRACT}

Background: With the follow-up extending to 5 years, the outcomes of SYNTAX (Synergy Between Percutaneous Coronary Intervention with TAXUS and Cardiac Surgery) trial were comparable between coronary artery bypass graft $(\mathrm{CABG})$ and percutaneous coronary intervention (PCI) in left-main (LM) patients with intermediate SYNTAX scores of 23-32. A subdivision depending on SYNTAX score will help to identify unsuitable LM patients with intermediate SYNTAX scores to receive PCI treatment.

Methods: Between January 2011 and June 2013, 104 patients with LM Coronary Artery Disease (CAD) undergoing PCI were selected retrospectively. We compared clinical outcomes in patients with SYNTAX score $<27$ and $\geq 27$. The follow-up time was $25.23 \pm 7.92$ months. Kaplan-Meier survival analyses and Cox proportional hazards models were used to compare various outcomes between two groups.

Results: Higher rates of repeated revascularization $(18.2 \%$ versus $4.2 \%, P=.027)$ and major adverse cerebrocardiovascular events (MACCE) (24.2\% versus $7.0 \%$, $P=.014$ ) were shown in patients with SYNTAX score $\geq 27$. After multivariate adjustment, a significant higher risk of repeated revascularization (hazard ratio: 6.25, 95\% confidence interval: 1.48 to $26.37, P=.013$ ) and MACCE (hazard ratio: $4.49,95 \%$ confidence interval: 1.41 to $14.35, P=.011$ ) were also found in patients with SYNTAX score $\geq 27$.

Conclusions: Based on the higher rate of repeated revascularization and MACCE, patients with LM CAD and intermediate SYNTAX scores will need a subdivision to identity the one not benefit from PCI. CABG is still the standard treatment method for patients of LM CAD with a SYNTAX score of $\geq 27$.

Received December 12, 2016; received in revised form August 7, 2017; accepted August 20, 2017.

Correspondence: Chunxiao Zhang MD, Beijing Anzhen Hospital, Capital Medical University, Peking University International Hospital, No.2 Anzhen Road, Chaoyang District, Beijing, China; (+86) 153-0101-8588; (E-mail: chunxiaozhangmd@outlook.com).

\section{INTRODUCTION}

The SYNTAX (Synergy Between Percutaneous Coronary Intervention With TAXUS and Cardiac Surgery) score was developed using a combination of several previously validated angiographic classifications that aimed to grade the coronary artery anatomy with respect to the number of lesions and their functional impact, location, and complexity[Sianos 2005]. The prognostic utility of the SYNTAX score has been recently validated in different settings, including in patients with three-vessel [Valgimigli 2007; Lemesle 2009] or left main (LM) coronary artery disease (CAD) [Birim 2009; Capodanno 2009] that underwent either percutaneous coronary intervention (PCI) or coronary artery bypass grafting (CABG). However, the SYNTAX score was originally conceived as an aid to decision making for PCI patient selection. From this perspective, how to distinguish patients who will most benefit from PCI is of clinical interest.

With the follow-up extending to 5 years, the SYNTAX trial demonstrated that a SYNTAX score of $\geq 33$ is associated with higher risk of major adverse cerebro-cardiovascular events (MACCE) after PCI [Kappetein 2011; Morice 2014]. The difference for patients with intermediate SYNTAX scores (23-32) between CABG and PCI further increased, with the exception of those patients with LM CAD, where outcomes between CABG and PCI were comparable [Kappetein 2011]. In this study, we focused on identifying which patients in the subgroup of LM CAD with intermediate SYNTAX scores were unsuitable to be treated by PCI by developing a new threshold point for intermediate SYNTAX scoring.

\section{MATERIALS AND METHODS}

\section{Study population}

From January 2011 to June 2013, all consecutive patients with multi-vessel CAD involving LM undergoing PCI at our center were enrolled retrospectively into a registry database. The inclusive criteria were: 1 . Age of patients was greater than 18 years old; 2 . The target vessels were no less than two major branches of the coronary arteries, such as LAD, right 
Table 1. Clinical Demographic Characteristics of Patients

\begin{tabular}{|c|c|c|c|}
\hline & $\begin{array}{c}\text { SYNTAX } \\
\text { Score }<27(71)\end{array}$ & $\begin{array}{c}\text { SYNTAX } \\
\text { Score } \geq 27(33)\end{array}$ & $P$ \\
\hline Age (years)* & $60.96 \pm 9.74$ & $58.79 \pm 10.0$ & .27 \\
\hline Female gender & $8(11.3 \%)$ & $2(6.1 \%)$ & .50 \\
\hline Pre-MI & $32(45.1 \%)$ & $12(36.4 \%)$ & .40 \\
\hline Pre-PCl & $9(12.7 \%)$ & $3(9.1 \%)$ & .75 \\
\hline Diabetes & $15(21.1 \%)$ & $8(24.2 \%)$ & .72 \\
\hline Hypertension & $42(59.2 \%)$ & $17(51.5 \%)$ & .46 \\
\hline Hyperlipoidemia & $35(49.3 \%)$ & $14(42.4 \%)$ & .51 \\
\hline Family history of CAD & $4(5.6 \%)$ & $3(9.1 \%)$ & .68 \\
\hline Pre-CVD & $4(5.6 \%)$ & $1(3.0 \%)$ & .54 \\
\hline PVD & $1(1.4 \%)$ & $1(3.0 \%)$ & .54 \\
\hline Smoking history & $35(49.3 \%)$ & $14(42.4 \%)$ & .51 \\
\hline \multicolumn{4}{|l|}{ Location of lesions } \\
\hline RCA & $67(94.4 \%)$ & $27(81.8 \%)$ & .04 \\
\hline LCX & $46(64.8 \%)$ & $23(69.7 \%)$ & .62 \\
\hline PLAD & $63(88.7 \%)$ & $28(84.8 \%)$ & .75 \\
\hline Three-vessel disease & $44(62.0 \%)$ & $18(54.5 \%)$ & .47 \\
\hline $\operatorname{LVEF}(\%)^{*}$ & $58.60 \pm 10.35$ & $60.40 \pm 11.01$ & .32 \\
\hline Total number of lesions* & $2.58 \pm 0.50$ & $4.27 \pm 0.57$ & $<.001$ \\
\hline Total number of stents* & $2.62 \pm 0.70$ & $3.82 \pm 1.01$ & $<.001$ \\
\hline Pre-operation TIMI* & & & .96 \\
\hline 0 & $5(7.0 \%)$ & $3(9.1 \%)$ & \\
\hline 1 & $4(5.6 \%)$ & $2(6.1 \%)$ & \\
\hline 2 & $4(5.6 \%)$ & $1(3.0 \%)$ & \\
\hline 3 & $58(81.7 \%)$ & $27(81.8 \%)$ & \\
\hline
\end{tabular}

MI, myocardial infarction; $\mathrm{PCl}$, percutaneous coronary intervention; $\mathrm{CAD}$, coronary artery disease; CVD, cerebrovascular disease; PVD, peripheral vascular disease; RCA, right coronary artery; LCX, left circumflex coronary artery; PLAD, proximal left anterior descending coronary artery; LVEF, left ventricular ejection fraction; TIMI, TIMI grade of blood flow. *Wilcoxon rank sum test.

coronary artery (RCA), left circumflex artery (LCX) and left main branch (LM); 3. One of the target vessels was LM; 4. The SYNTAX score was between 20 and 35.104 patients were selected from this database.

\section{Procedural and post-intervention practices}

The procedural choice was made by the consensus of the cardiologists and cardiac surgeons in the study group. The details of the treatment would be revealed to the patients. Then, a final authorization from the patients and their family would be obtained. The interventional strategy, as well as the choice of the various devices and the administration of therapies during the procedure, was left to the operator's discretion and standard practice. After the procedure, patients treated with DES were prescribed Clopidogrel for at least 1 year. Aspirin was prescribed for all patients daily.

\section{SYNTAX score calculation}

The total SYNTAX score was derived from the summation of the individual scorings for each separate lesion (defined as $\geq 50 \%$ stenosis in vessel $\geq 1.5 \mathrm{~mm}$ ). Full details on SYNTAX score calculation were reported elsewhere [Sianos 2005]. All angiographic variables pertinent to SYNTAX score calculation were computed by 2 of 3 experienced cardiologists who were blinded to procedural data and clinical outcome on angiograms was obtained. In case of disagreement, the opinion of the third observer was obtained, and the final decision was made by consensus.

\section{Clinical outcomes and follow up}

The primary endpoints of this study included cardiac death; postoperative myocardial infarction based on new Q-waves or troponin I levels of 5 times greater than normal value; repeated revascularization including all target and nontarget vessels; clinically evident stroke; stent thrombosis; and the composite end points of all of the above (major adverse cerebra-cardiovascular events (MACCE) [Cutlip 2007; Kip 2008]. The secondary endpoints included cardiac readmission and consistent chest pain after PCI. Also, the length of stay in hospital and total cost for medical care in hospital were evaluated. All clinical follow-up data was collected by direct telephone interviews.

\section{Statistical analysis}

According to the previous studies, a SYNTAX score of 27 was chosen as the cut-off point. Divided by the SYNTAX score $<27$ and $\geq 27$ [Capodanno 2009], patient characteristics were compared between the two groups. Differences in continuous variables between subgroups of patients were evaluated using Student's unpaired $t$ tests or Wilcoxon rank sum nonparametric tests as appropriate. The normality assumption for continuous variables was evaluated by the KolmogorovSmirnov test. Categorical variables were presented as counts and percentages. The differences in categorical variables between patient subgroups were evaluated with chi-square test or Fisher exact test. The cumulative rates of primary endpoints were estimated by the Kaplan-Meier method, and the log-rank test was used to evaluate differences between groups.

To reduce the effect of selection bias and potential confounding in this observational study, the outcome parameter was adjusted by means of a Cox multivariable proportional hazard regression model with the propensity score, which was calculated from variables deemed clinically relevant or statistically significant in the univariate analysis, such as age, gender, previous myocardial infarction (MI), previous PCI, diabetes, hypertension (DBP > 90 and/or SBP > 140), hyperlipoidemia, family history of coronary artery disease (CAD), previous cerebrovascular disease (CVD), peripheral vascular disease (PVD), smoking history, left ventricular ejection fraction (LVEF), total number of stents, TIMI grade of blood flow, and location of the lesions, including right coronary artery (RCA), left circumflex coronary artery (LCX), and 


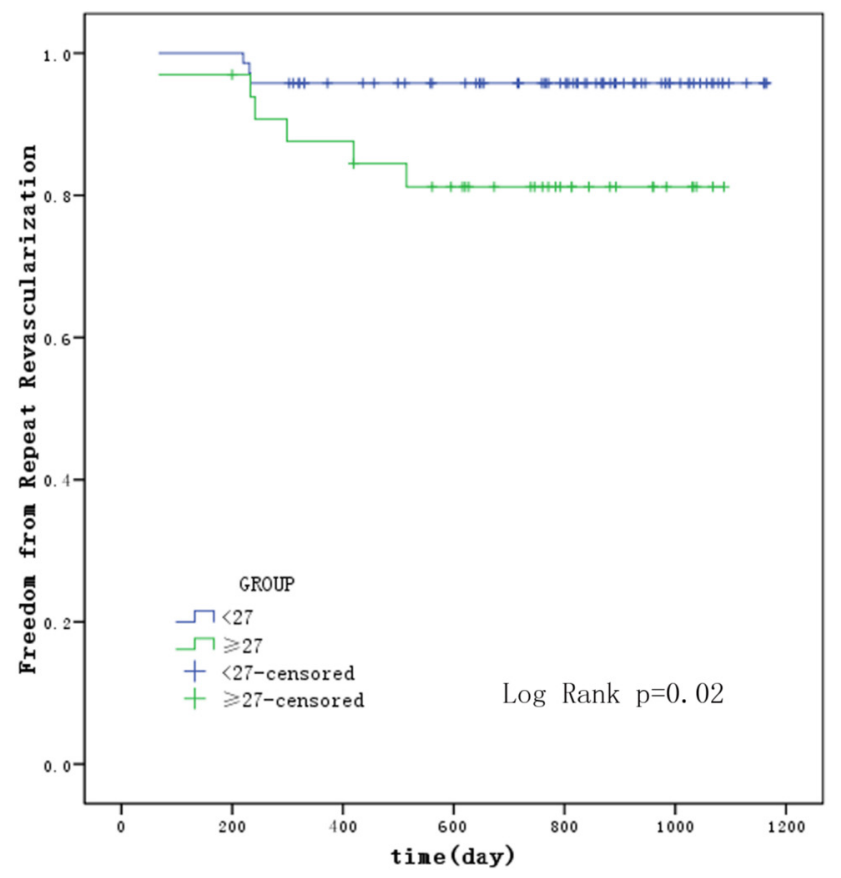

Figure 1. Kaplan-Meier survival analyses used to compare repeated revascularization between the two groups.

proximal left anterior descending coronary artery (PLAD). The interaction effect of a SYNTAX score $\geq 27$ was determined by a Cox proportional hazard regression analysis with the investigated cut-off point and adjusting propensity score entered into the model. For all analyses, a 2 -sided value of $P<.05$ was considered statistically significant. All data was processed using the Statistical Package for Social Sciences version 15 (SPSS Inc., Chicago, Illinois).

\section{RESULTS}

Baseline clinical and angiographic characteristics of the study population were roughly similar between two groups (Table 1 ). The mean age was $60.27 \pm 9.83$ years old. The number of males in this study group was 94 (90.4\%). The overall SYNTAX score in the study population did not demonstrate a normal distribution $(P<.001)$ but a distinct right skewness, ranging from 20 to 35 with a median of 25 (interquartile range, 22 to 32 ) and a mean of $26.77 \pm 6.32$. Mean of SYNTAX score was $23.35 \pm 3.32$ in lower risk group (SYNTAX score $<27)$ and $30.71 \pm 3.12$ in higher risk group $($ SYNTAX score $\geq 27)(P<.001)$.

\section{Early clinical outcomes and hospitalization costs}

There were no mortalities or complications during the postoperative period before discharged in either group. The length of stay (LOS) in hospital was similar between two groups $(P=.40)$. Cost of medical care in hospital between two groups also showed non-significant difference $(P=.57)$ (Table 2).

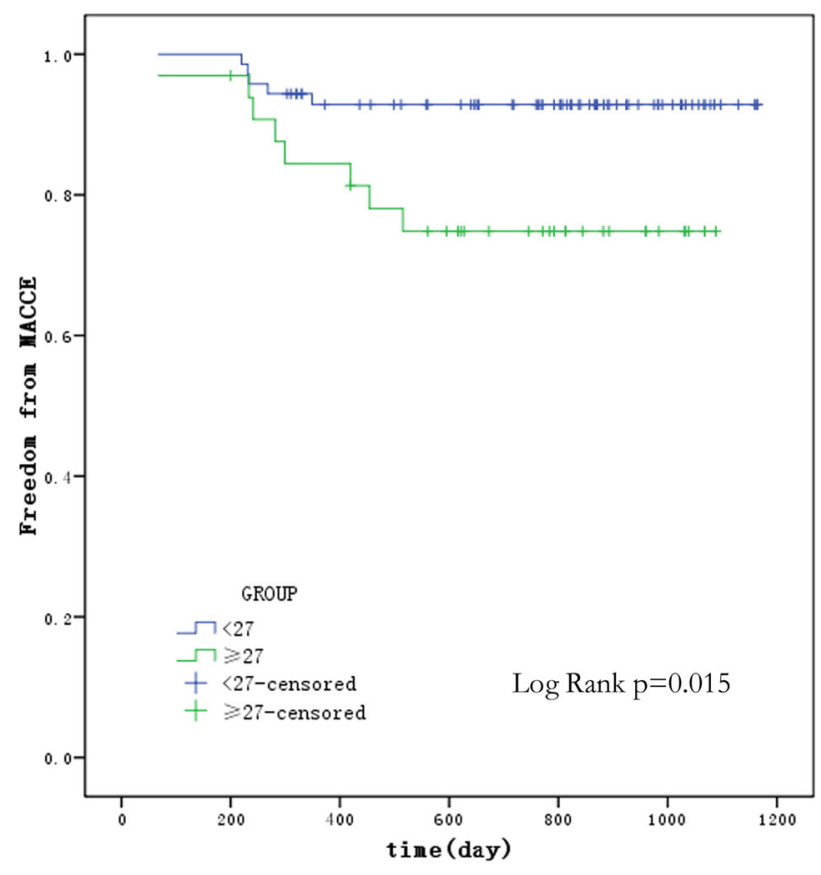

Figure 2. Kaplan-Meier survival analyses used to compare MACCE between two groups.

\section{Follow-up outcomes}

No patients were lost in follow-up. A mean duration was $25.23 \pm 7.92$ months (ranging 6 to 35 months based on the date of enrollment). As shown in Table 2, there were no deaths in either group. One patient (3.0\%) suffered from post-MI was reported in group with SYNTAX score $\geq 27$, and this patient received revascularization in our institute. There were two patients reported as clinically evident stroke in each group (SYNTAX score $\geq 27$ group, 6.1\% and SYNTAX score $<27$ group, $2.8 \% ; P=.59$ ). Also, no patients with late stent thrombosis were found in either group. A significant difference was shown between two groups when comparing the repeated revascularization and MACCE. The rates of repeated revascularization were $4.2 \%$ in group with SYNTAX score $<27$ and $18.2 \%$ in group with SYNTAX score $\geq 27(P=.03)$. The rates of MACCE between two groups were mainly driven by the cases of repeat revascularization and showed significant difference as well (SYNTAX score $<27$ group, $7.0 \%$; and SYNTAX score $\geq 27$ group, $24.2 \% ; P=.01)$.

In the survival analysis, the difference between two groups was shown in Kaplan-Meier curves. The repeated revascularization mostly occurred from 6 months to 18 months when followed up. The results of log rank test were significant for repeated revascularization $(P=.02)$ (Figure 1$)$ and MACCE $(P=.015)$ (Figures 1, 2). After adjustment for potential confounders, the results of Cox proportional hazard regression analysis were also showed statistical significance for repeated revascularization and MACCE $(\mathrm{HR}=$ 4.49, 95\% CI, 1.41-14.35, $P=.01$ for MACCE; $\mathrm{HR}=6.25$, $95 \% \mathrm{CI}, 1.48-26.37, P=.01$ for repeated revascularization; Table 3). 
Table 2. Postoperative Outcomes

\begin{tabular}{lccc}
\hline & $\begin{array}{c}\text { SYNTAX score } \\
<27(71)\end{array}$ & $\begin{array}{c}\text { SYNTAX score } \\
\geq 27(33)\end{array}$ & $P$ \\
\hline Mortality & $0(0 \%)$ & $0(0 \%)$ & NS \\
Postoperative MI & $0(0 \%)$ & $1(3.0 \%)$ & 0.32 \\
Repeat revascularization & $3(4.2 \%)$ & $6(18.2 \%)$ & 0.03 \\
Stroke & $2(2.8 \%)$ & $2(6.1 \%)$ & 0.59 \\
Stent thrombosis & $0(0 \%)$ & $0(0 \%)$ & NS \\
MACCE & $5(7.0 \%)$ & $8(24.2 \%)$ & 0.01 \\
Cardiac readmission & $14(19.7 \%)$ & $9(27.3 \%)$ & 0.39 \\
Chest pain & $30(42.3 \%)$ & $12(36.4 \%)$ & 0.57 \\
Hospital LOS (d)* & $7.31 \pm 3.52$ & $6.61 \pm 3.39$ & 0.40 \\
Total hospital cost (¥)* & $62465.16 \pm$ & $67452.92 \pm$ & 0.57 \\
& 21891.65 & 27550.09 & \\
\hline
\end{tabular}

*Wilcoxon rank sum test. MI, myocardial infarction; MACCE, major adverse cerebro-cardiovascular events; LOS, length of stay; Cl, confidence interval; NS, nonsignificant.

\section{DISCUSSION}

The present study showed that in the intermediate SYNTAX scores group of patients with LM CAD, the presence of a SYNTAX score $\geq 27$ was associated with a higher risk of MACCE and repeated revascularization after PCI. After adjustment for potential confounders, patients with SYNTAX score $\geq 27$ treated with PCI had almost 5-fold increase in the likelihood of about 2-year MACCE and 6-fold increase in repeated revascularization comparing with the patients with SYNTAX score $<27$. Overall, these findings support that in the intermediate SYNTAX scores group of patients with LM $\mathrm{CAD}$, a threshold of 27 is clinically useful to select unsuitable ones who will not be benefit from PCI treatment.

The SYNTAX trial has demonstrated that CABG remained the standard care for patients with three-vessel or LM CAD due to the lower rates of the combined end points of MACCE at 1 year [Serruys 2009]. With follow-up extending to 3 years, the Kaplan-Meier curve of MACCE after PCI or CABG in patients with low SYNTAX scores $(\leq 22)$ remained superimposed. In patients with intermediate SYNTAX scores (23-32), there was no significant difference at 1 year, but the diverging curves during follow-up suggested that CABG may be of greater benefit in these patients. For patients with SYNTAX scores $\geq 33$, the difference between CABG and PCI further increased during follow-up, demonstrating the superiority of CABG compared to PCI in this subgroup. Detailed separate analyses of patients with LM and three-vessel disease demonstrated similar findings, except for patients with intermediate SYNTAX scores of 23-32, where outcomes between CABG and PCI were comparable. Here the difference between CABG and PCI seems negligible in patients with LM disease. In those with three-vessel disease,
Table 3. Results of Cox Proportional Hazards Ratios for Different Outcomes Between the Two Groups

\begin{tabular}{lccc}
\hline Group & $\mathrm{HR}^{*}$ & $95 \% \mathrm{Cl}$ & $P$ \\
\hline Repeat revascularization & 6.25 & $1.48-26.37$ & .01 \\
MACCE & 4.49 & $1.41-14.35$ & .01 \\
\hline
\end{tabular}

*Propensity score adjusted. MACCE, major adverse cerebro-cardiovascular events; $\mathrm{HR}$, hazard ratios; $\mathrm{Cl}$, confidence interval.

the rate of MACCE after CABG was significantly lower than after PCI [Kappetein 2011; Head 2014]. Some recent randomized clinical trials have compared CABG with PCI in the LM patients with intermediate SYNTAX score, but the results remained negligible [Park 2011; Farkouh 2012].

Insight into the LM CAD group of patients with intermediate SYNTAX score through this study, the outcomes of follow up was heterogeneous. In the patients above the threshold of 27, there were more MACCEs happened compared with the group below 27. The repeated revascularization was the major contribution to the difference between two groups. Higher rate of repeated revascularization has always been criticized for treatment with PCI in patients with complex CAD from the era of bare metal stent (BMS) [Venkitachalam 2009]. Although the application of drug eluting stent (DES) decreased the incidence, it has been still a difficult problem to be solved until now [Li 2009; Ben-Gal 2010; Park 2010; Takayama 2010]. As shown in this study, there was a subgroup of LM CAD patients with intermediate SYNTAX score who were not suitable for the PCI treatment because of the repeated revascularization. The lesions of the LM increased the difficulty and risk of PCI. This may be the main reason for the higher rate of repeated revascularization in this group of patients. Considering the updated LM revascularization guidelines have recently assigned a class IIb recommendation to PCI in patients with low/intermediate SYNTAX scores, the current US and European revascularization guidelines assign CABG a IA indication in most LM CAD patients with 1,2 , or $3 \mathrm{VD}$ with low, intermediate, or high SYNTAX scores [Hillis 2011; Kolh 2014] and the ongoing Evaluation of Xience Prime or Xience V versus CABG for Effectiveness of Left Main Revascularization (EXCEL) trial is enrolling LM patients with mild-to-moderate anatomic complexity (SYNTAX score $\leq 32$ ) using a more contemporary stent and current surgical techniques, it is worth sheding more light on the controversial issues of LM revascularization. Through this study, although there was not a control group of CABG with SYNTAX score $\geq 27$ comparing with the PCI, we can compare the results from a previous study that the outcomes of the CABG-treated LM CAD group with SYNTAX score $\geq 27$ were better [Holzhey2010]. The inference is that CABG is still the standard treatment method for patients with LM CAD in SYNTAX score $\geq 27$.

\section{Study limitations}

As a preliminary study, the observed study design and the sample size were limitations for this study. It only showed 
a major trend for further study. A random clinical trial will be necessary. Also, we need to compare the PCI and CABG directly between the subgroup of SYNTAX score of 27-32 in future studies.

\section{CONCLUSIONS}

This study demonstrated that not all LM patients with intermediate SYNTAX scores will benefit from treatment of PCI. A threshold of SYNTAX score of 27 will be helpful to identifying the unsuitable patients undergoing PCI treatment. Because of the higher rate of repeated revascularization, CABG will be still the standard treatment method for LM CAD patients with intermediate and high SYNTAX scores.

\section{ACKNOWLEDGMENTS}

This work was supported by the Foundation of Beijing Anzhen Hospital, Capital Medical University (No. 2013Z04, No. 2016P02). The work was carried out at Beijing Anzhen Hospital, Capital Medical University, Beijing, China.

\section{REFERENCES}

Ben-Gal Y, Moses JW, Mehran R, et al. 2010. Surgical versus percutaneous revascularization for multivessel disease in patients with acute coronary syndromes: analysis from the ACUITY (Acute Catheterization and Urgent Intervention Triage Strategy) trial. JACC Cardiovasc Interv 3:1059-1067.

Birim O, van Gameren M, Bogers AJ, et al. 2009. Complexity of coronary vasculature predicts outcome of surgery for left main disease. Ann Thorac Surg 87:1097-1104; discussion 1104-1095.

Capodanno D, Di Salvo ME, Cincotta G, et al. 2009. Usefulness of the SYNTAX score for predicting clinical outcome after percutaneous coronary intervention of unprotected left main coronary artery disease. Circ Cardiovasc Interv 2:302-308.

Cutlip DE, Windecker S, Mehran R, et al. 2007. Clinical end points in coronary stent trials: a case for standardized definitions. Circulation 115:2344-2351.

Farkouh ME DM, Sleeper LA, Siami FS, et al. 2012. Strategies for multivessel revascularization in patients with diabetes. N Engl J Med $367: 2375-2384$.

Head SJ, Farooq V, Serruys PW, Kappetein AP. 2014. The SYNTAX score and its clinical implications. Heart 100:169-177.

Hillis LD, Smith PK, Anderson JL, et al. 2011. 2011 ACCF/AHA Guideline for Coronary Artery Bypass Graft Surgery. A report of the American College of Cardiology Foundation/American Heart Association Task Force on Practice Guidelines. Developed in collaboration with the American Association for Thoracic Surgery, Society of Cardiovascular Anesthesiologists, and Society of Thoracic Surgeons. J Am Coll Cardiol 58:e123-210.
Holzhey DM, Luduena MM, Rastan A, et al. 2010. Is the SYNTAX score a predictor of long-term outcome after coronary artery bypass surgery? Heart Surg Forum13:E143-148.

Kappetein AP1 FT, Mack MJ, Morice MC, et al. 2011. Comparison of coronary bypass surgery with drug-eluting stenting for the treatment of left main and/or three-vessel disease: 3-year follow-up of the SYNTAX trial. Eur Heart J 32:2125-2134.

Kip KE, Hollabaugh K, Marroquin OC, Williams DO. 2008. The problem with composite end points in cardiovascular studies: the story of major adverse cardiac events and percutaneous coronary intervention. J Am Coll Cardiol 51:701-707.

Lemesle G, Bonello L, de Labriolle A, et al. 2009. Prognostic value of the Syntax score in patients undergoing coronary artery bypass grafting for three-vessel coronary artery disease. Catheter Cardiovasc Interv 73:612-617.

Li Y, Torguson R, Syed AI, et al. 2009. Effect of drug-eluting stents on frequency of repeat revascularization in patients with unstable angina pectoris or non-ST-elevation myocardial infarction. Am J Cardiol 104:1654-1659.

Morice MC SP, Kappetein AP, Feldman TE, et al. 2014. Five-Year Outcomes in Patients With Left Main Disease Treated With Either Percutaneous Coronary Intervention or Coronary Artery Bypass Grafting in the Synergy Between Percutaneous Coronary Intervention With Taxus and Cardiac Surgery Trial. Circulation 129:2388-2394.

Park DW, Kim YH, Yun SC, et al. 2010. Long-term outcomes after stenting versus coronary artery bypass grafting for unprotected left main coronary artery disease: 10-year results of bare-metal stents and 5-year results of drug-eluting stents from the ASAN-MAIN (ASAN Medical Center-Left MAIN Revascularization) Registry. J Am Coll Cardiol 56:1366-1375

Park SJ, Kim YH, Park DW, et al. 2011. Randomized trial of stents versus bypass surgery for left main coronary artery disease. N Engl J Med 364:1718-1727.

Serruys PW, Morice MC, Kappetein AP, et al. 2009. Percutaneous coronary intervention versus coronary-artery bypass grafting for severe coronary artery disease. N Engl J Med 360:961-972.

Sianos G, Morel MA, Kappetein AP, et al. 2005. The SYNTAX Score: an angiographic tool grading the complexity of coronary artery disease. EuroIntervention 1:219-227.

Takayama T, Hiro T, Hirayama A. 2010. Is angioplasty able to become the gold standard of treatment beyond bypass surgery for patients with multivessel coronary artery disease? Circ J 74:2744-2749.

Valgimigli M SP, Tsuchida K, Vaina S, et al. 2007. Cyphering the complexity of coronary artery disease using the syntax score to predict clinical outcome in patients with three-vessel lumen obstruction undergoing percutaneous coronary intervention. Am J Cardiol 99:1072-1081.

Venkitachalam L, Kip KE, Selzer F, et al. 2009. Twenty-year evolution of percutaneous coronary intervention and its impact on clinical outcomes: a report from the National Heart, Lung, and Blood Institute-sponsored, multicenter 1985-1986 PTCA and 1997-2006 Dynamic Registries. Circ Cardiovasc Interv 2:6-13. 